\title{
OBSERVATIONS ON THE TREATMENT OF RHEUMATIC FEVER WITH VITAMIN $P$
}

\author{
BY
}

\section{J. F. RINEHART}

\section{University of California Medical School, San Francisco}

Recently, in this journal (1943), the writer reviewed evidence pertaining to the possible rôle of nutritional deficiency as a contributory factor in the aetiology of rheumatic fever. In this report it was shown that a series of experimental, epidemiological and clinical considerations implicated vitamin $\mathrm{C}$ deficiency as a factor in the pathogenesis of rheumatic fever. While vitamin $\mathrm{C}$ deficiency may be important in predisposing to the initial attack of rheumatic fever and may, per se, be of some value in treatment, ascorbic acid has failed to exert any marked influence on the course of the illness or to prevent recurrences.

In 1941 we became interested in the possible therapeutic value of vitamin $\mathbf{P}$ in rheumatic fever and, in the paper referred to, reported on the apparent favourable influence of this substance in 3 cases of persistently active rheumatic fever. Subsequently, we recorded preliminary observations on small numbers of cases of rheumatic fever so treated (1944). Vitamin $P$ was so designated because of its reputed influence on capillary permeability. In the excellent original report of Armentano and his colleagues (1936), evidence was presented that this substance was effective in correcting the abnormal capillary fragility in cases of vascular purpura as well as restoring to normal the increased permeability of capillaries encountered in certain infections. It is of interest that most cases of so-called vascular purpura probably have an allergic basis. Considerable evidence suggests the operation of allergic factors in rheumatic fever and the frequent occurrence of haemorrhagic manifestations is well known. The plant pigment or flavone constituting vitamin $\mathbf{P}$ is considered to act in conjunction with ascorbic acid. In view of these considerations and the early encouraging observations made in treatment of rheumatic fever it is natural that we should consider it desirable to extend investigations in this field.

It is the purpose of this paper briefly to report observations which we have made on 39 cases of rheumatic fever which have been treated with vitamin $\mathbf{P}$ for periods of one month or longer. A high proportion of the cases have been of the polycyclic or refractory type. All showed activity of the rheumatic process at the time treatment was instituted, as evidenced by the sedimentation rate The diagnosis of rheumatic fever has been established beyond reasonable question in all cases. In many an accelerated sedimentation rate was the only clear evidence of continued activity of the disease. Inasmuch as sedimentation rates had been determined by different methods, for purposes of analysis and graphic representation, it was necessary to establish appropriate equivalents for the several methods applied. While we have not been able to establish absolutely accurate comparative scales, they are entirely adequate for the purposes. In most cases a single method was used in individual cases.

\section{Observations}

Analysis of the data reveals the following. Of the 39 cases, 24 were children and 15 were adults. Twenty-six of the 39 cases had exhibited persistent activity for periods of 6 weeks or longer in spite of the application of the usual methods of management. The average duration of the illness in this group was 10 weeks. The average sedimentation rate of the 39 cases at the time treatment was instituted was $33 \mathrm{~mm}$. per hour in terms of the Wintrobe scale. One month from the institution of treatment the average sedimentation rate was $17.5 \mathrm{~mm}$. per hour. Thirty-four of the 39 cases exhibited significant slowing of the sedimentation rate at the end of 6 weeks (4 weeks in 12 of the cases in which observation was terminated at the time). Two cases showed no significant changes in sedimentation rate; in 3 the rate of sedimentation was more rapid. Two of the latter suffered obvious intercurrent infection which would account for this. Between 4 and 6 weeks after therapy 22 of the 39 cases showed either no evidence of activity or minimal activity.

The findings in the 26 persistent cases are of particular interest. In this group the average initial sedimentation rate was $32 \mathrm{~mm}$. per hour and at the end of 1 month was $15.5 \mathrm{~mm}$. per hour (Wintrobe scale). It is noteworthy that this occurred in such a short period of time in a group of cases that, under observation, had shown persistent activity over considerable periods of time. At the end of 1 month, 22 of the 26 cases in this 


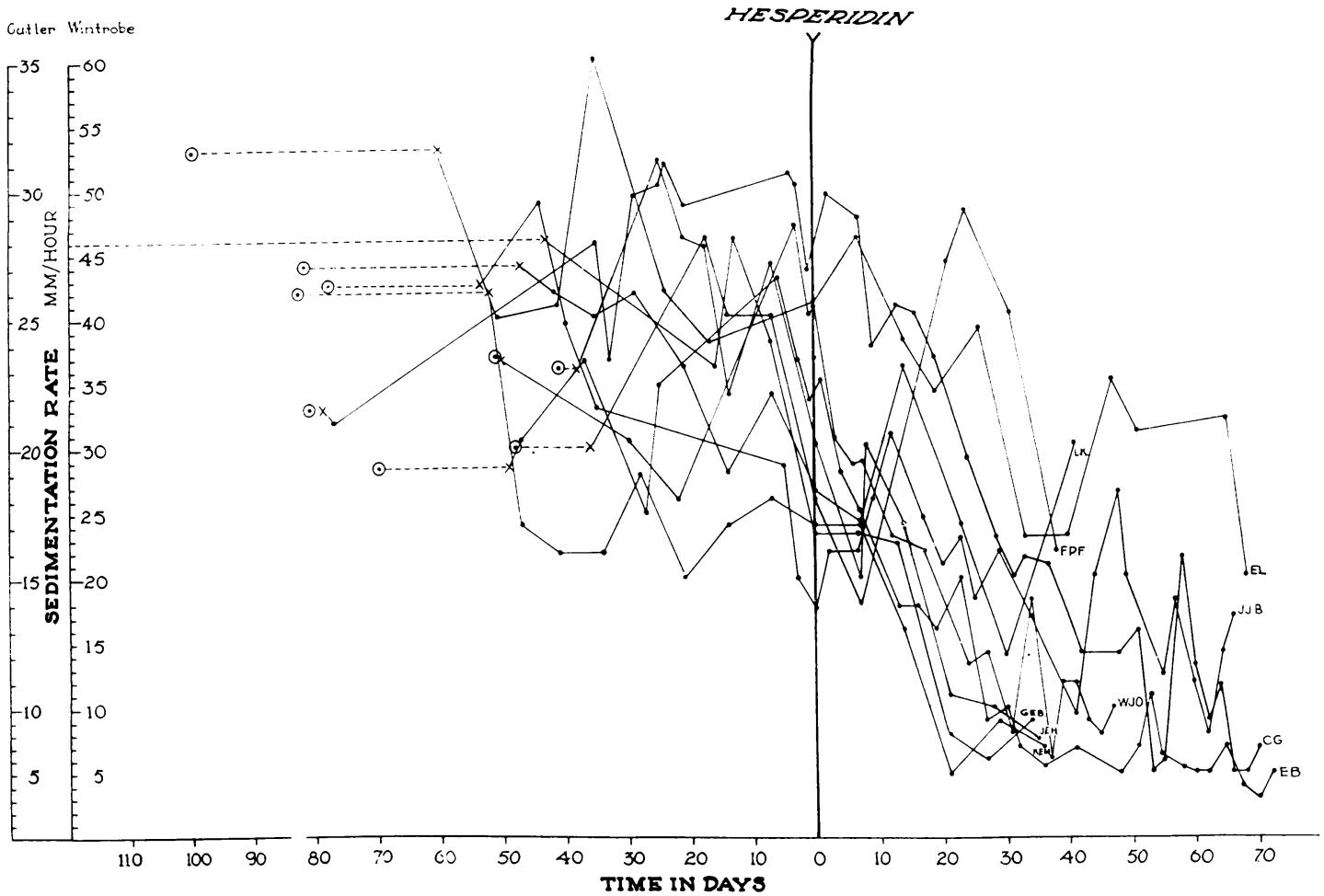

FIG. 1.-Curves of sedimentation rate in 16 cases of persistent rheumatic fever in children treated with hesperidin. The circles indicate time of clinical onset; the crosses indicate the first recorded sedimentation rate and the dots indicate subsequent determination.

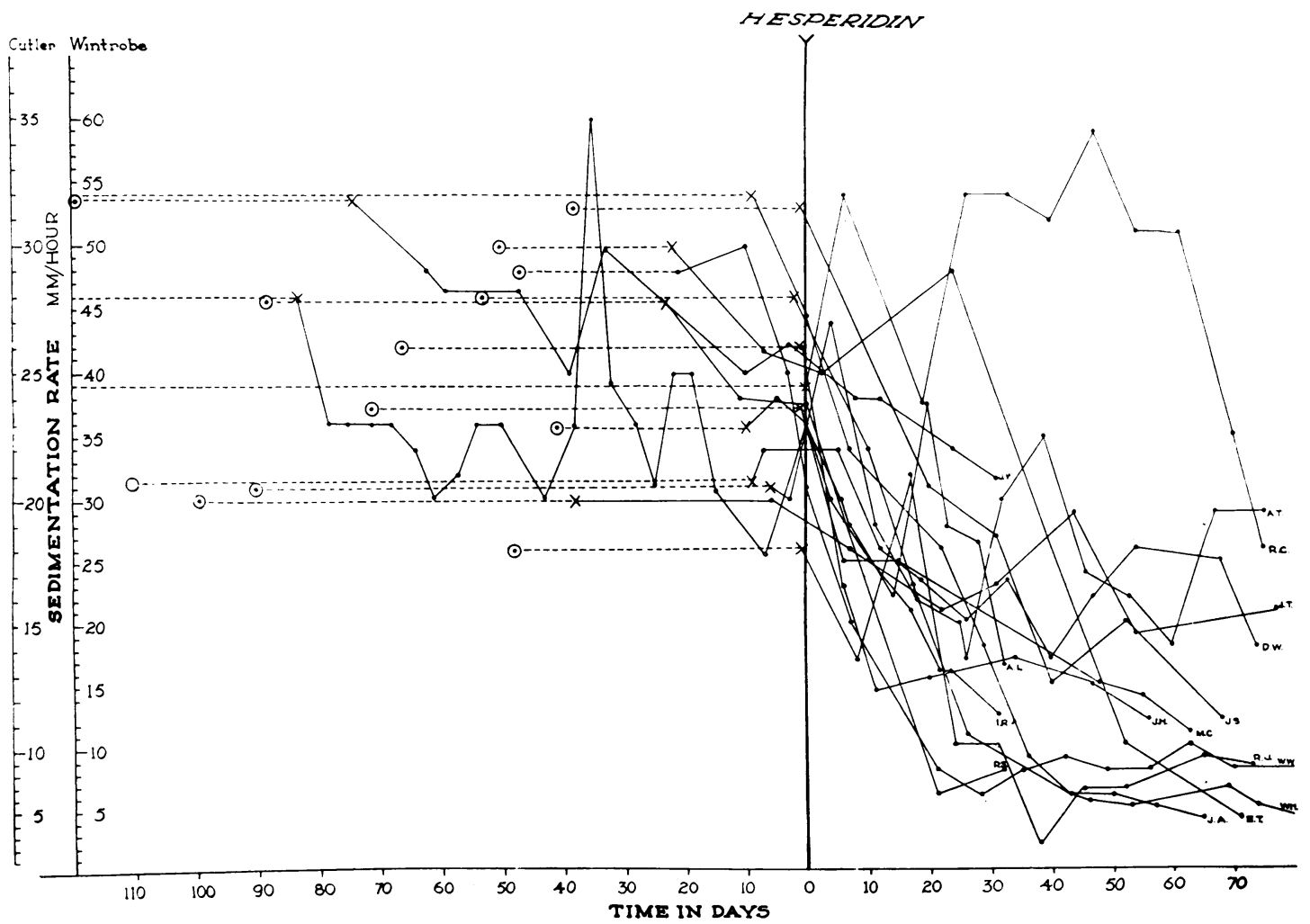

FIG. 2.-Curves of sedimentation rate in 10 adult cases of persistent rheumatic fever treated with hesperidin. Symbols as in Fig. 1. 
group had exhibited significant slowing of the sedimentation rate. In 3 the rate was somewhat more rapid and in 1 it was unchanged. Sixteen cases showed either minimal or no activity at the end of one month. The graphic representation of the sedimentation rates shown in Figs. 1 and 2, suggests most strongly that improvement was related to the treatment.

Emphasis has been placed upon the sedimentation rate in this study because it is the most critical objective index of activity and gauge of progress in cases in which the diagnosis of rheumatic fever is established. In many it was the only definite evidence of continued active disease. Insofar as other manifestations of activity existed, improvement occurred which paralleled the slowing sedimentation rate.

\section{Discussion}

It is considered highly probable by the writer that the slowing of the sedimentation rate in the cases reported is related to the treatment. This is believed to be so particularly in the persistent cases where clinical observations indicated prolonged activity prior to treatment. It will be noted by reference to Figs. 1 and 2 that many cases showed significant slowing of the sedimentation rate within 2 weeks. The cases of relatively short duration, although showing similar responses, are less convincing in that spontaneous recovery is frequently observed in a 4 to 6 week period. Although the persistent cases act to some extent as their own control, it is naturally desirable to extend the studies utilizing alternate cases as controls. This is being done at the present time, and the data now available indicates that the treated cases are showing more favourable response than the untreated controls.

It should be pointed out that in the cases studied, vitamin $\mathbf{P}$ was given as additional therapy, i.c. it was added to the therapeutic regime of the patient at the time study of the case was undertaken. Although it has not been studied, we have no evidence that the substance possesses analgesic or antipyretic properties analogous to salicylates. The vitamin P-containing material which we have used in most of the cases is crude Hesperidin which has been fortified with Hesperidin Methyl Chalcone.* It is prepared in compressed tablets containing 0.5 grams of crude Hesperidin and $20 \mathrm{mg}$. of Hesperidin Methyl Chalcone. The usual dosage has been 1.5 grams; one tablet is given three times daily with meals. No toxic or ill effects have been observed which are ascribable to this medication. In a number of cases the material has been administered for several months. The mode of action is uncertain. If vitamin $\mathrm{P}$ acts as a co-enzyme with vitamin C as suggested by Szent-Györgyi (1939), deficiency of this factor would block effective utilization of ascorbic acid. Also, it is of interest that the cases of vascular purpura which are reported as benefited by vitamin $\mathbf{P}$ are generally considered to be of allergic nature. There is considerable evidence that an allergic mechanism operates in rheumatic fever.

In view of the great importance of rheumatic fever as a cause of cardiac disability and death, studies of promise should be pursued with vigour. If nutritional deficiency is a contributory aetiologic factor, it is evident that prevention would be feasible.

\section{Summary}

Observations are recorded in 39 cases of rheumatic fever treated with vitamin P. Evidence is presented that this substance exerts a beneficial influence on the course of the illness as reflected chiefly by slowing the sedimentation rate. Extension of studies on the application of vitamin $\mathbf{P}$ in treatment and prophylaxis of rheumatic fever are indicated.

The writer gratefully acknowledges the cooperation of Dr. Helen Johnson, Chief of the Cardiac Programme of the Crippled Children's Bureau of the California State Department of Public Health.

\section{REFERENCES}

Armentano, L., Bentsáth, A., Béres, T., Rusznyák, Stefan, and Szent-Györgyi, Albert (1935). Dtsche med. Wschr., 1936, 36, 1325 .

Rinehart, J. F. (1943). Ann. rheum. Dis., 3, 154

- and Johnson Helen (1944). J. Clin. Invest, 23, 941.

Szent-Györgyi, Albert von (1939). “ On Oxidation, Fermentation, Vitamins, Health and Disease." Baltimore, Maryland.

* We are indebted to The Abbott Laboratories for liberal supplies of the fortified Crude Hesperidin. 